\title{
Towards Certifiable Advanced Flight Control Systems, A Sensor Based Backstepping Approach
}

\author{
W. Falkena*, E. R. van Oort ${ }^{\dagger}$ and Q. P. Chu ${ }^{\ddagger}$ \\ ASTI, Kluyverweg 1, Delft, 2629HS, the Netherlands
}

\begin{abstract}
This paper uses singular perturbation theory in order to design a backstepping control system. Earlier applications using singular perturbation theory for control of non-affine systems exist, but here the focus is shifted to uncertain systems. Dealing with uncertainties in this way could be a major benefit for certification of advanced control laws. The reason for this is twofold. First, the backstepping construct, using Lyapunov functions, guarantees the stability of the controlled system. Second, by using measurements of the state derivatives rather than relying on perfect knowledge of the system, a major drawback of backstepping is removed: the need for adaptation to uncertain parameters or unknown model structure. As this method bears resemblance to sensor based nonlinear dynamic inversion, the control law developed in this paper will be referred to as sensor based backstepping. Preliminary results for systems with uncertainty and sensor noise look very promising and future investigation into the effects of time-delays is needed.
\end{abstract}

\section{Introduction}

Design of advanced control systems using neural networks, ${ }^{1}$ dynamic inversion, ${ }^{2}$ backstepping, ${ }^{3,4}$ etc., has been ongoing in the aerospace sector for quite some time. Certification and implementation of these control laws has proven not to be easy, however. DO-178B or Software Considerations in Airborne Systems and Equipment Certification has been accepted by the FAA for certification of airborne software. In short it stipulates that the flight control algorithms should work with a probability of failure of $10^{-9}$, or once in a billion flying hours. ${ }^{5}$ To achieve such a low probability, the control law must be able to handle model uncertainty, sensor noise, time delays and preferably actuator failure or even structural damage throughout the full extent of the flight envelope.

Backstepping control is deemed a good candidate for a certifiable advanced control law, due to it's use of Lyapunov functions, that guarantees system stability. However, conventional backstepping is sensitive to model uncertainties. This problem can be approached from two sides. Robust backstepping incorporates specific uncertainties in the controller design, such that stability can be guaranteed even in the presence of these uncertainties. Alternatively, adaptive backstepping incorporates parameter estimation in the backstepping controller to adapt to parameter uncertainties or even failures. ${ }^{6}$ However, as mentioned by Jacklin, ${ }^{7}$ giving the control system the power to make rapid automatic adjustments to enable self-healing in case of failures, also carries the potential that it makes a healthy aircraft unflyable in case of software malfunction.

This paper uses singular perturbation theory, Tikhonov's Theorem and a backstepping construct in order to control uncertain systems. Hovakimyan et al. proposed a controller for non-affine systems based on Tikhonov's Theorem in 2007. ${ }^{8}$ Here a similar controller is used, but with the perspective shifted to uncertain systems. Dealing with uncertainties in this way could be a major benefit for certification of advanced control laws. The reason for this is twofold. First, the backstepping construct, using Lyapunov functions, guarantees the stability of the controlled system. Second, by using measurements of the state derivatives rather than relying on perfect knowledge of the system, the need for adaptation to uncertain parameters or unknown model structure is removed. As this method bears resemblance to sensor based

\footnotetext{
*Scientific Researcher, Delft University of Technology - Control and Simulation - Aerospace Software and Technology Institute, Kluyverweg 1 2629HS Delft the Netherlands.

${ }^{\dagger}$ PhD student, Delft University of Technology - Control and Simulation, Kluyverweg $12629 H S$ Delft the Netherlands.

$¥$ Associate Professor, Delft University of Technology - Control and Simulation, Kluyverweg $12629 \mathrm{HS}$ Delft the Netherlands.
} 
nonlinear dynamic inversion, ${ }^{9}$ the developed control law will be referred to as Sensor Based Backstepping $(\mathrm{SBB})$ in this paper.

This paper is structured as follows. First the singular perturbation theory will be summarized in Section II, followed by the controller design of Hovakimyan et al. in Section III. Section IV provides simple examples, demonstrating the advantages of the new method, and Section $\mathrm{V}$ contains a particularly useful example of controlling the attitude rate of an aircraft by measuring the angular acceleration. Conclusions and recommendations are given at the end of this paper, in Section VI.

\section{Singular Perturbation Theory}

Singular perturbations, i.e., small perturbations that can not be approximated as zero, cause time-scale separated behavior in systems that contain slow and fast transients in the reaction of the system to an external input. ${ }^{3}$ Consider a singularly perturbed nonlinear system with the following state space description:

$$
\begin{aligned}
\dot{x} & =f(t, x, u, \epsilon), \quad x(0)=\xi(\epsilon) \\
\epsilon \dot{u} & =g(t, x, u, \epsilon), \quad u(0)=\eta(\epsilon)
\end{aligned}
$$

where $\xi$ and $\eta$ depend smoothly on the small positive parameter $\epsilon$. Assume that $f$ and $g$ are continuously differentiable in their arguments for $(t, x, u, \epsilon) \in[0, \infty] \times D_{x} \times D_{u} \times\left[0, \epsilon_{0}\right]$, where $D_{x} \in \mathbb{R}^{n}, D_{u} \in \mathbb{R}^{m}$ are domains and $\epsilon_{0}>0$. In addition, let the system (1) be in standard form, i.e. $0=g(t, x, u, 0)$ has $k \geq 1$ isolated real roots $u=h_{i}(t, x), i \in\{1, \ldots, k\}$ for each $(t, x) \in[0, \infty] \times D_{x}$. To obtain the $i$-th model, the roots can be substituted in (1) at $\epsilon=0$,

$$
\dot{x}=f(t, x, h(t, x), 0), x(0)=\xi(0)
$$

System (2) is also referred to as the reduced system. In singular perturbation theory, the system given by,

$$
\frac{d v}{d \tau}=g(t, x, v+h(t, x), 0), v(0)=\eta_{0}-h\left(0, \xi_{0}\right)
$$

is called the boundary layer system, where $v(t, x)=u-h(t, x)$, the normal time-scale $t$ is replaced by the new time-scale $\tau=\frac{t}{\epsilon}$ and $\eta_{0}=\eta(0)$ and $\xi_{0}=\xi(0),(t, x) \in[0, \infty) \times D_{x}$ are treated as fixed parameters. Then the following result is due to Tikhonov, ${ }^{3,10}$

Theorem 1. Consider the singular perturbation system (1) and let $u=h(t, x)$ be an isolated root of $g(t, x, u, 0)$. Assume that the following conditions hold for all $(t, x, u-h(t, x), \epsilon) \in[0, \infty] \times D_{x} \times D_{v} \times\left[0, \epsilon_{0}\right]$ for some domains $D_{x} \in \mathbb{R}^{n}$ and $D_{v} \in \mathbb{R}^{m}$, which contain the corresponding origins.

(a) On any compact subset of $D_{x} \times D_{v}$ the function $f, g$, their first partial derivatives with respect to $(x, u, \epsilon)$, and the first partial derivative of $g$ with respect to $t$ are continuous and bounded, $h(t, x)$ and $\frac{\partial g}{\partial u}(t, x, u, 0)$ have bounded first derivatives with respect to their arguments, $\frac{\partial f}{\partial x}(t, x, h(t, x))$ is Lipschitz in $x$ uniformly in $t$, and the initial conditions for $\xi$ and $\eta$ are smooth functions of $\epsilon$.

(b) The origin is an exponentially stable equilibrium point of the reduced system (2). There exists a Lyapunov function $V:[0, \infty) \times D_{x} \rightarrow[0, \infty)$ that satisfies

$$
\begin{aligned}
& W_{1}(x) \leq V(t, x) \leq W_{2}(x) \\
& \frac{\partial V(t, x)}{\partial t}+\frac{\partial V(t, x)}{\partial x} f(t, x, h(t, x), 0) \leq-W_{3}(x)
\end{aligned}
$$

for all $(t, x) \in[0, \infty) \times D_{x}$, where $W_{1}, W_{2}$, and $W_{3}$ are continuous positive definite functions on $D_{x}$. Let $c$ be a nonnegative number such that $x \in D_{x} \mid W_{1}(x) \leq c$ is a compact subset of $D_{x}$.

(c) The origin is an equilibrium point of the boundary layer system (3), which is exponentially stable uniformly in $(t, x)$.

Let $R_{v} \in D_{v}$ denote the region of attraction of the autonomous system,

$$
\frac{d v}{d \tau}=g\left(0, \xi_{0}, v+h\left(0, \xi_{0}\right), 0\right)
$$

and let $W_{v}$ be a compact subset of $R_{v}$. Then for each compact set $W_{x} \in x \in D_{x} \mid W_{2}(x) \leq \rho c, 0<\rho<1$, there exists a positive constant $\epsilon_{*}$ such that for all $t \geq 0, \xi_{0} \in W_{x}, \eta_{0}-h\left(0, \xi_{0}\right) \in W_{v}$ and $0<\epsilon<\epsilon_{*}$. 
System (1) has a unique solution $x_{\epsilon}$ on $[0, \infty)$ and $x_{\epsilon}(t)-x_{00}(t)=O(\epsilon)$ holds uniformly for $t \in[0, \infty)$, where $x_{00}(t)$ denotes the solution of the reduced system.

In other words given a sufficiently time-scale separated nonlinear system $\left(0<\epsilon<\epsilon_{*}\right)$, that describes physical phenomena (Theorem 1.a) and with stabilizable slow dynamics (Theorem 1.b and Theorem 1.c), then the fast dynamics will be stable and remain near the slow dynamic behavior of the system $\left(x_{\epsilon}(t)-x_{00}(t)=O(\epsilon)\right)$.

\section{Controller Design Based on Fast Dynamics}

Hovakimyan et al. propose a controller based on the time-scale separation properties of a singularly perturbed system. ${ }^{8}$ Consider the nonlinear system,

$$
\dot{x}=f(t, x, u)
$$

where $x(0)=x_{0}$ for $(x, u) \in D_{x} \times D_{u}$ and where $D_{x} \subset \mathbb{R}^{n}$ and $D_{u} \subset \mathbb{R}^{m}$ are domains that contain the corresponding origins. Here $x$ denotes the state vector and $u$ the input vector. The function $f$ is continuously differentiable in its arguments. Furthermore assume $\partial f / \partial u$ is bounded away from zero for $(x, u) \in \Omega_{x, u} \subset D_{x} \times D_{u}$, where $\Omega_{x, u}$ is a compact set of possible initial conditions, i.e., there exists a $b_{0}>0$ such that $\partial f / \partial u>b_{0}$.

A reference signal $y_{r}$ is defined for the state $x$ and the tracking error between the state and this reference is given by, $e=x-y_{r}$. Therefore, the error dynamics of the system can be written as,

$$
\dot{e}=f\left(t, e+y_{r}, u\right)-\dot{y}_{r}
$$

A controller for (5) can be constructed based on the following fast dynamics,

$$
\epsilon \dot{u}=-\operatorname{sgn}\left(\frac{\partial f}{\partial u}\right) \mathbf{f}(t, e, u)
$$

where $\mathbf{f}(t, e, u)$ is the mapping $D_{e} \times D_{u} \rightarrow \mathbb{R}^{m}$. Application of Theorem 1 leads to the following result.

Theorem 2. Assume that the following conditions hold for all $(t, e, u-h(t, e), \epsilon) \in[0, \infty] \times D_{e} \times D_{v} \times\left[0, \epsilon_{0}\right]$ for some domains $D_{e} \in \mathbb{R}^{n}$ and $D_{v} \in \mathbb{R}^{m}$, which contain the corresponding origins.

(a) On any compact subset of $D_{e} \times D_{v}$ the function $f$ and the first partial derivatives with respect to $(e, u, t)$, are continuous and bounded, $h(t, e)$ and $\frac{\partial f}{\partial u}(t, e, u)$ have bounded first derivatives with respect to their arguments, $\frac{\partial f}{\partial e}(t, e, h(t, e))$ is Lipschitz in $e$, uniformly in $t$.

(b) The origin is an exponentially stable equilibrium point of the reduced system,

$$
\dot{e}=f\left(t, y_{r}, h(t, 0)\right)
$$

The mapping $e \rightarrow f\left(e+y_{r}, h(t, e)\right)$ is continuously differentiable and Lipschitz in e, uniformly in $t$.

(c) The mapping $(t, e, v) \rightarrow \frac{\partial f}{\partial u}(t, e, v+h(t, e))$ is bounded from below by some positive number for all $(t, e) \in[0, \infty] \times D_{e}$

Then the origin of the boundary layer system,

$$
\frac{d v}{d \tau}=-\operatorname{sgn}\left(\frac{\partial f}{\partial u}\right) \mathbf{f}(t, e, v+h(t, e))
$$

is exponentially stable. Moreover, let $\Omega_{v}$ be a compact subset of $R_{v}$, where $R_{v} \subset D_{v}$ denotes the region of attraction of the autonomous system,

$$
\frac{d v}{d \tau}=-\operatorname{sgn}\left(\frac{\partial f}{\partial u}\right) \mathbf{f}\left(0, e_{0}, v+h\left(0, e_{0}\right)\right)
$$

Then for each compact subset $\Omega_{e} \subset D_{e}$, there exists a positive constant $\epsilon_{*}$ such that for all $t \geq 0, e_{0} \in$ $\Omega_{e}, u_{0}-h\left(0, e_{0}\right) \in \Omega_{v}$, and $0<\epsilon<\epsilon_{*}$. System (4),(6) has a unique solution $x_{\epsilon}$ on $[0, \infty)$ and $x_{\epsilon}(t)=$ $y_{r}(t)+O(\epsilon)$ holds uniformly for $t \in[T, \infty)$.

In other words given a sufficiently time-scale separated nonlinear system $\left(0<\epsilon<\epsilon_{*}\right)$, that describes physical phenomena (Theorem 2.a), is minimum phase (Theorem 2.b) and controllable (Theorem 2.c), then the fast dynamics will be stable and follow the reference signal $\left(x_{\epsilon}(t)=y_{r}(t)+O(\epsilon)\right)$. The advantage of using the stabilizing controller described in Equation (6) becomes clear in the following sections. 


\section{Simple Example}

Consider the system,

$$
\dot{x}=h(u)=f+g u
$$

with $x \in \mathbb{R}^{n}, u \in \mathbb{R}^{m}$ and $f$ and $g$ constants. Again the tracking error, $e=x-y_{r}$, is introduced. A backstepping controller can be designed using the positive definite, radially unbounded, Control Lyapunov Function $V(e)=\frac{1}{2} e^{2}$, of which the derivative should be negative definite for asymptotic stability in the sense of Lyapunov,

$$
\begin{aligned}
\dot{V}(e) & =e \dot{e}=e\left(\dot{x}-\dot{y}_{r}\right)=e\left(f+g u-\dot{y}_{r}\right) \\
u & =g^{-1}\left(-c_{1} e+\dot{y}_{r}-f\right)
\end{aligned}
$$

where $c_{1}$ is a design parameter. If $c_{1}>0$, then $\dot{V}(e)$ will be negative definite for the control law $(9)$ and hence the stability of the closed-loop system is guaranteed.

Using the theory described in Section III, it is possible to design a different controller, that uses measurements of $\dot{x}$ rather than knowledge about $f$. A controller can be designed based on the following fast dynamics,

$$
\epsilon \dot{u}=-\operatorname{sgn}\left(\frac{\partial h}{\partial u}\right)\left[\dot{x}-\dot{x}_{d e s}\right]
$$

where $\dot{x}_{d e s}$ denotes the desired state derivatives, which can be constructed using the derivative of $V(e)$,

$$
\begin{aligned}
\dot{V}(e) & =e \dot{e}=e\left(\dot{x}_{d e s}-\dot{y}_{r}\right) \\
\dot{x}_{d e s} & =-c_{2} e+\dot{y}_{r}
\end{aligned}
$$

with $c_{2}>0$ to render $\dot{V}(e)$ negative definite. The controller therefore becomes,

$$
\epsilon \dot{u}=-\operatorname{sgn}(g)\left[\dot{x}+c_{2}\left(x-y_{r}\right)-\dot{y}_{r}\right]
$$

and is referred to as SBB in the remainder of this paper. The system (7),(12) clearly satisfies the assumptions of Theorem 2.a and 2.c. For compliance to assumption 2.b the exponential stability of the equilibrium point of the reduced system needs to be demonstrated. The isolated root, $\dot{u}=0$, can be written as,

$$
0=-\operatorname{sgn}(g)\left[\dot{x}+c_{2}\left(x-y_{r}\right)-\dot{y}_{r}\right]=\dot{e}+c_{2}\left(x-y_{r}\right)
$$

leading to the reduced system definition,

$$
\dot{e}(t, x, h(x, t), 0)=-c_{2}\left(x-y_{r}\right)
$$

with equilibrium point $x_{e q}=y_{r}$. Application of Lyapunov's direct method (Theorem 4.5 in Murray et $a .^{11}$ ) shows exponential stability of the reduced system, thereby verifying Theorem 2.b. The controller in (12) should stabilize the system.

The top row in Figure 1 shows the response of the system (7) to the different controllers. Note that the initial response, from zero to two seconds, of the SBB controlled system is caused by starting the control actions when the system is not in a trimmed condition. Adding a the trim input, $u_{0}=g^{-1}(-f)$, such that $\dot{x}(0)=0$, results in the response shown in the bottom row of Figure 1 .

The advantage of using SBB becomes clear in the presence of uncertainty. Increasing $f$ by $10 \%$ leads to the response shown in the top row of Figure 2. Increasing $g$ by $10 \%$ leads to the response shown in the bottom row of Figure 2. The normal backstepping controller needs some time to stabilize the error dynamics, thereby creating a large steady state error. The SBB controller only uses the sign of $\partial h / \partial u$ and is therefore unaffected by the parameter changes.

A slightly more complex system can be written as,

$$
\dot{x}=f x^{a}+g u
$$

with $x \in \mathbb{R}^{n}, u \in \mathbb{R}^{m}, a \in \mathbb{R}$ and $f$ and $g$ constants. Again it is possible to construct a backstepping controller as,

$$
u=g^{-1}\left(-c_{3} e+\dot{y}_{r}-f\left(e+y_{r}\right)^{a}\right)
$$

Since the SBB controller does not make use of $f(x)$, it remains the same. 
Figure 3 shows the response of the system (15) to the different controllers with and without uncertainty in the nonlinearity. The top row shows that perfect tracking is obtained for both controllers when the system is nonlinear $(a=2)$ and the controller knows this nonlinearity. The bottom row shows the response of the system (15) when linearity $(a=1)$ is used in the controllers, while the system actually behaves nonlinearly. The SBB controller still tracks perfectly, whereas the conventional backstepping method is notably influenced by the assumption of linearity.

\section{Application to the Moment Equation}

As shown in the previous section, using SBB has an advantage over conventional backstepping when dealing with uncertainties. A typical application in which SBB may be used is the attitude rate control of an aircraft. Consider the moment equation, ${ }^{12}$

$$
\dot{\omega}=J^{-1}\left(M_{\text {aero }}+M_{\text {control }}-\omega \times J \omega\right)
$$

where $\omega=[p, q, r]^{T}$ are the rotational rates of the aircraft in body reference frame, $J$ is the inertia tensor, $M_{\text {aero }}$ are the aerodynamic moments acting on the aircraft except for the moments due to control surface deflections and $M_{\text {control }}$ are the moments due to control surface deflections. The former of these moments may be written as,

$$
M_{\text {aero }}=\bar{q} S C_{1}\left(C_{2}+\frac{C_{1}}{2 V_{T}} C_{\omega} \omega\right)
$$

where $\bar{q}$ is the dynamic pressure, $S$ the wing area and,

$$
C_{1}=\left[\begin{array}{ccc}
b & 0 & 0 \\
0 & \bar{c} & 0 \\
0 & 0 & b
\end{array}\right], C_{\omega}=\left[\begin{array}{ccc}
C_{l_{p}} & 0 & C_{l_{r}} \\
0 & C_{m_{q}} & 0 \\
C_{n_{p}} & 0 & C_{n_{r}}
\end{array}\right] \text { and } C_{2}=\left[\begin{array}{c}
C_{l_{\beta}} \beta+C_{l_{\dot{\beta}}} \dot{\beta} b /\left(2 V_{T}\right) \\
C_{m_{0}}+C_{m_{\alpha}} \alpha+C_{m_{\dot{\alpha}}} \dot{\alpha} \bar{c} /\left(2 V_{T}\right) \\
C_{n_{\beta}} \beta+C_{n_{\dot{\beta}}} \dot{\beta} b /\left(2 V_{T}\right)
\end{array}\right]
$$

with $V_{T}$ the true airspeed, $b$ the wing span, $\bar{c}$ the mean aerodynamic chord, $C_{i}$ the aerodynamic moment coefficients due to the body angular rates $\omega$, the angle of attack $\alpha$, the rate of change of angle of attack $\dot{\alpha}$ and the side slip angle $\beta$. In order to relate $\alpha, \beta$ and their derivatives to the body rotational rates, the equations of motion in the wind axis system can be used. An expression for these equations of motions are found in Aircraft Control and Simulation, ${ }^{13}$

$$
\begin{aligned}
\dot{V}_{T} & =\frac{1}{m}[\cos \alpha \cos \beta \sin \beta \cos \beta \sin \alpha] F_{b} \\
\dot{\alpha} & =q-(p \cos \alpha+r \cos \alpha) \tan \beta+\frac{1}{m} \frac{1}{V_{T} \cos \beta}[-\sin \alpha 0 \cos \alpha] F_{b} \\
\dot{\beta} & =p \sin \alpha-r \cos \alpha+\frac{1}{m} \frac{1}{V_{T}}[-\cos \alpha \sin \beta \cos \beta \sin \alpha \sin \beta] F_{b}
\end{aligned}
$$

where,

$$
F_{b}=\left[\begin{array}{c}
F_{x, b} \\
F_{y, b} \\
F_{z, b}
\end{array}\right]
$$

It is assumed that the effect of the forces $F_{b}$ on $\dot{\alpha}$ and $\dot{\beta}$ is small in comparison to the rotational rates and are therefore neglected in the controller design. Equation (18) can now be adjusted to,

$$
M_{\text {aero }}=\bar{q} S C_{1}\left(C_{3}+C_{\text {aero }}[\alpha \beta]^{T}+\frac{C_{1}}{2 V_{T}}\left(C_{\omega}+C_{\omega, \text { aero }}\right) \omega\right)
$$

with,

$$
\begin{aligned}
C_{3}= & {\left[\begin{array}{c}
0 \\
C_{m_{0}} \\
0
\end{array}\right], C_{\text {aero }}=\left[\begin{array}{cc}
0 & C_{l_{\beta}} \\
C_{m_{\alpha}} & 0 \\
0 & C_{n_{\beta}}
\end{array}\right] \text { and } } \\
C_{\omega, \text { aero }}= & {\left[\begin{array}{ccc}
C_{l_{\dot{\beta}}} \sin \alpha & -C_{l_{\dot{\beta}}} \cos \alpha \\
-C_{m_{\dot{\alpha}}} \cos \alpha \tan \beta & C_{m_{\dot{\alpha}}} & -C_{m_{\dot{\alpha}}} \cos \alpha \tan \beta \\
C_{n_{\dot{\beta}}} \sin \alpha & 0 & -C_{n_{\dot{\beta}}} \cos \alpha
\end{array}\right] }
\end{aligned}
$$



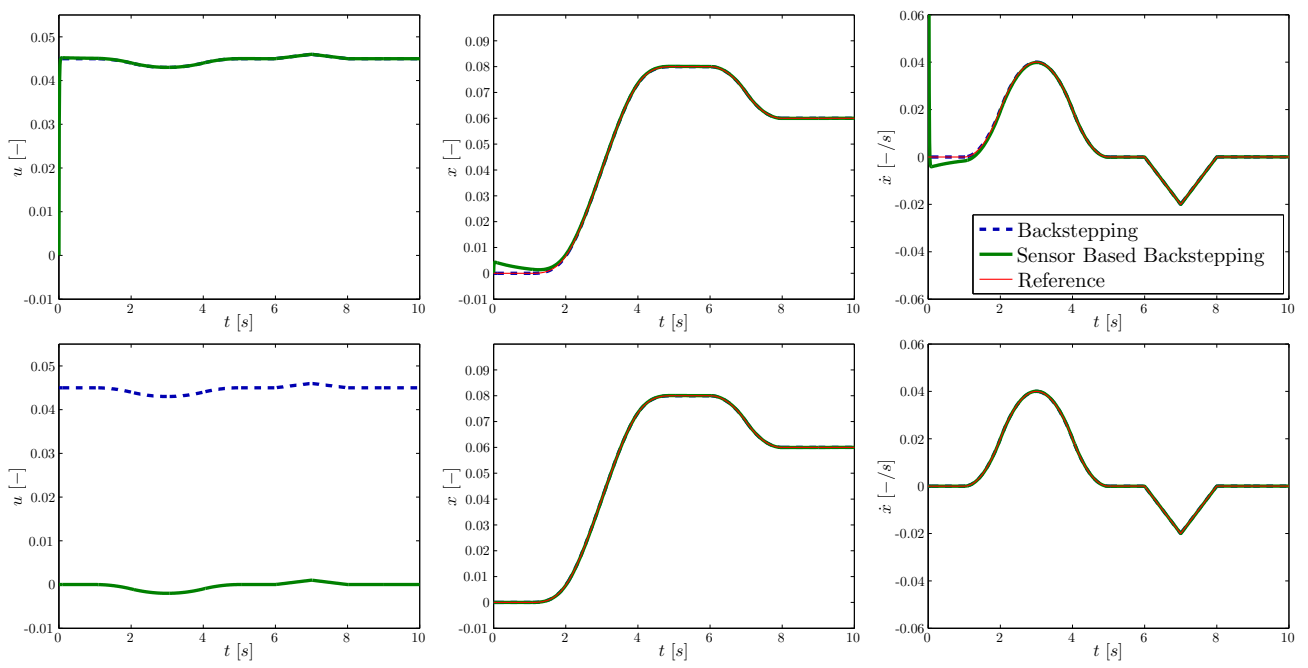

Figure 1. Response of system (7) using a backstepping $\left(c_{1}=1\right)$ and a sensor based backstepping controller $\left(c_{2}=1, \epsilon=0.1\right)$ without trim input (top row) and with trim input (bottom row).
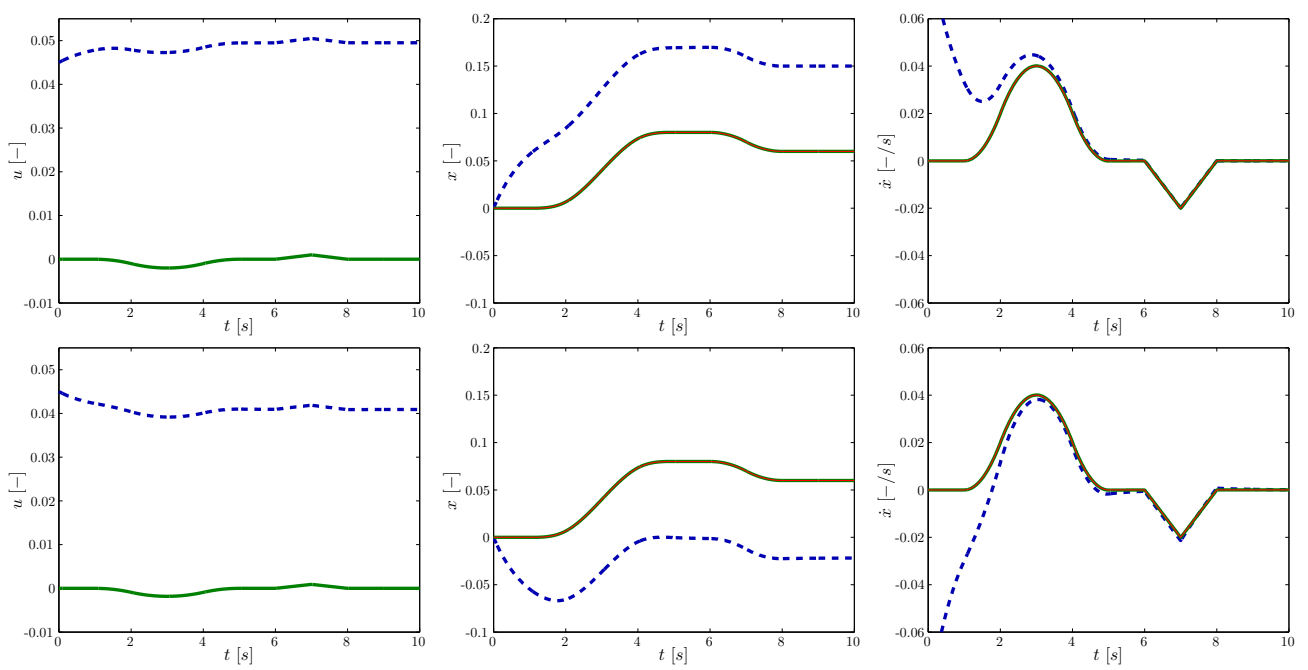

Figure 2. Response of system (7) using a backstepping $\left(c_{1}=1\right)$ and a sensor based backstepping controller $\left(c_{2}=1, \epsilon=0.1\right)$ with trim input in the presence of uncertainty in $f$ (top row) and $g$ (bottom row).
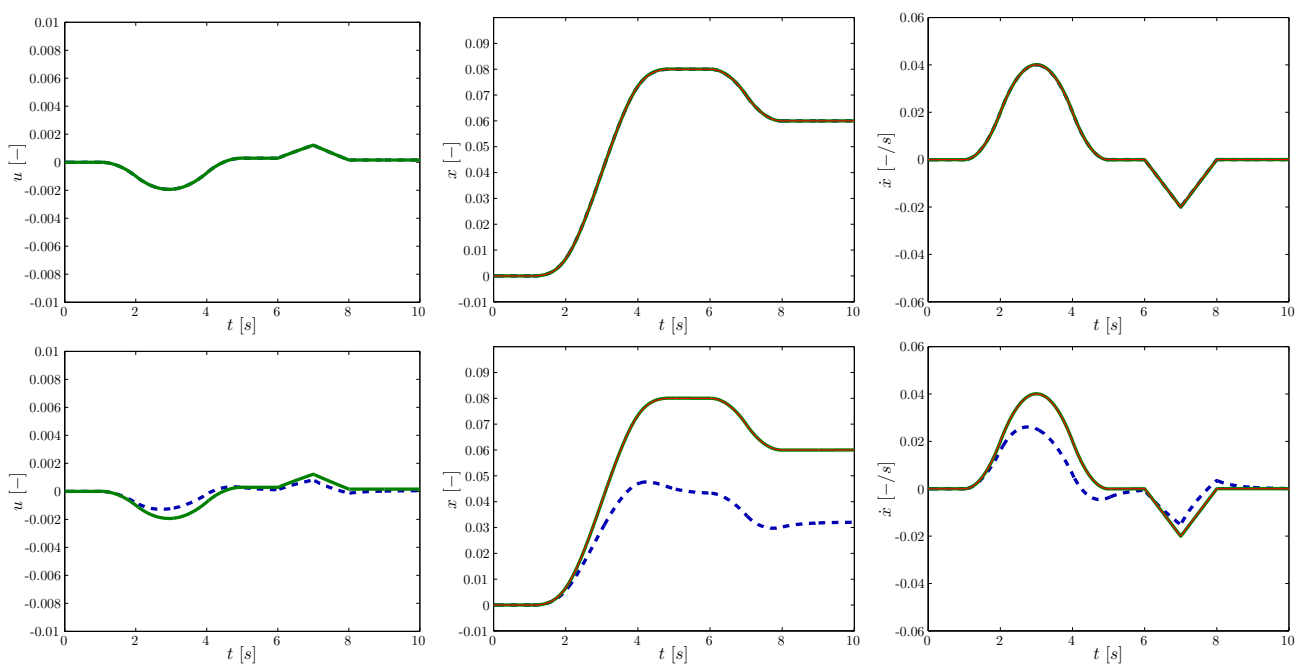

Figure 3. Response of system (15) with $a=2$ using a backstepping $\left(c_{3}=1\right)$ and a sensor based backstepping controller $\left(c_{2}=1, \epsilon=0.1\right.$ ) with $a=2$ (top row) and $a=1$ (bottom row). 
The latter of the two moments in Equation (17) may be written in similar fashion as,

$$
M_{\text {control }}=\bar{q} S C_{1} C_{u} u
$$

where,

$$
C_{u}=\left[\begin{array}{ccc}
C_{l_{\delta_{a}}} & 0 & C_{l_{\delta_{r}}} \\
0 & C_{m_{\delta_{e}}} & 0 \\
C_{n_{\delta_{a}}} & 0 & C_{n_{\delta_{r}}}
\end{array}\right] \text { and } u=\left[\begin{array}{c}
\delta_{a} \\
\delta_{e} \\
\delta_{r}
\end{array}\right]
$$

with $C_{u}$ the aerodynamic moment coefficients due to control surface deflection, also known as control surface effectiveness parameters and $\delta_{a, e, r}$ the aileron, elevator and rudder deflection angles.

Now we can simplify the notation of system (17) using the following functions,

$$
\begin{aligned}
\dot{x_{1}} & =\left[\begin{array}{ccc}
-\cos \alpha \tan \beta & 1 & -\cos \alpha \tan \beta \\
\sin \alpha & 0 & -\cos \alpha
\end{array}\right] x_{2} \\
\dot{x_{2}} & =J^{-1} \bar{q} S C_{1}\left(C_{3}+C_{\text {aero }} x_{1}+\frac{C_{1}}{2 V_{T}}\left(C_{\omega}+C_{\omega, \text { aero }}\left(x_{1}\right)\right) x_{2}+C_{u} u\right)-J^{-1}\left(x_{2} \times J x_{2}\right)
\end{aligned}
$$

where $x_{1}=\left[\begin{array}{ll}\alpha & \beta\end{array}\right]^{T}$ and $x_{2}=\omega$. In order to get some feeling for this system three consecutive 1 second singlets are given on the control surfaces. The system response is shown in Figure 4.
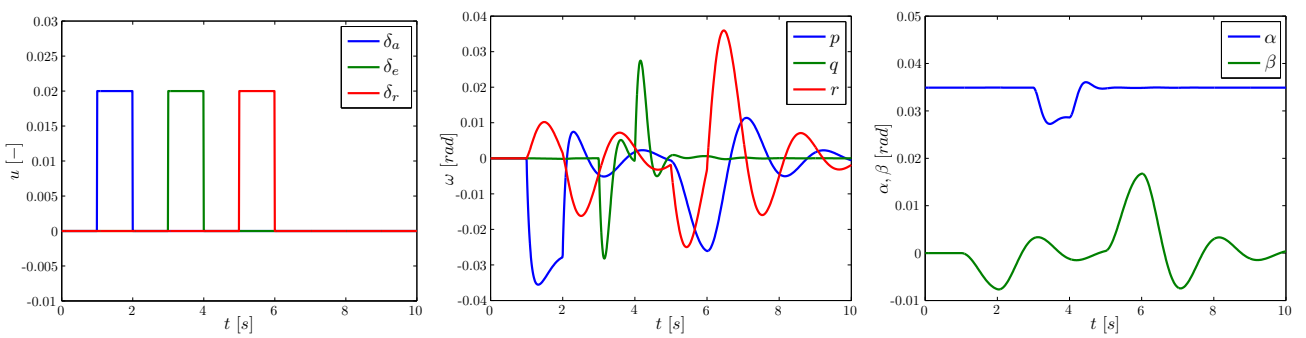

Figure 4. Response of system (27) when excited using 1 second singlets.

Again a backstepping controller will be designed. Using,

$$
\begin{aligned}
e & =x_{2}-y_{r} \\
V(e) & =\frac{1}{2} e^{2} \\
\dot{V}(e) & =e \dot{e}=e\left[J^{-1} \bar{q} S C_{1}\left(C_{3}+C_{a e r o} x_{1}+C_{\omega^{*}} x_{2}+C_{u} u\right)-J^{-1}\left(x_{2} \times J x_{2}\right)-\dot{y}_{r}\right] \\
u & =C_{u}^{-1}\left[\left(-C_{3}-C_{a e r o} x_{1}-C_{\omega^{*}}\left(e+y_{r}\right)\right)+J\left(C_{1} S \bar{q}\right)^{-1}\left(J^{-1}\left(x_{2} \times J x_{2}\right)+\dot{y}_{r}-c_{4} e\right)\right] \\
C_{\omega^{*}} & =\frac{C_{1}}{2 V_{T}}\left(C_{\omega}+C_{\omega, \text { aero }}\left(x_{1}\right)\right)
\end{aligned}
$$

choosing $c_{4}>0$ will render $\dot{V}(e)$ negative definite and therefore stabilizes the system.

The SBB controller that can be used is actually the same as the one in Equation (12). It is repeated here for convenience,

$$
\epsilon \dot{u}=-\operatorname{sgn}\left(\frac{\partial \dot{x}_{2}}{\partial u}\right)\left[\dot{x}_{2}-\dot{x}_{2, d e s}\right]=-\operatorname{sgn}\left(\frac{\partial \dot{x}_{2}}{\partial u}\right)\left[\dot{x}_{2}+c_{5}\left(x_{2}-y_{r}\right)-\dot{y}_{r}\right]
$$

with $c_{5}>0$ to stabilize the system. Evaluating the partial derivative of $\dot{x}_{2}$ to $u$ gives,

$$
\frac{\partial \dot{x}_{2}}{\partial u}=J^{-1} \bar{q} S C_{1} C_{u}
$$

Since $J, \bar{q}, S, C_{1}$ are all positive (definite), the only matrix of influence is the control effectiveness matrix $C_{u}$. The eigenvalues of this matrix are all negative when the direction of control surface deflections are defined as done by Mulder. ${ }^{12}$ This means $\operatorname{sgn}\left(\frac{\partial h}{\partial u}\right)$ will be -1 .

Figure 5 shows the response of the system (27) to the different controllers. The SBB controller is not fully able to track the system and a steady state error remains. The control inputs do not lead to instability of the system, however. The cause for this steady state error can be traced back to the internal dynamics, 
i.e., the influence of $\alpha$ and $\beta$. To improve the tracking behavior an integral term $\lambda=\int_{0}^{t} e d t$ can be added to the SBB controller,

$$
\begin{aligned}
V(e) & =\frac{1}{2} e^{2}+\frac{1}{2} k_{1} \lambda^{2} \\
\dot{V}(e) & =e \dot{e}+k_{1} \lambda e=e\left(\dot{x}_{d e s}-\dot{y}_{r}+k_{1} \lambda\right) \\
\dot{x}_{d e s} & =-c_{5} e+\dot{y}_{r}-k_{1} \lambda \\
\epsilon \dot{u} & =-\operatorname{sgn}\left(\frac{\partial \dot{x}_{2}}{\partial u}\right)\left[\dot{x}+c_{5}\left(x-y_{r}\right)-\dot{y}_{r}+k_{1} \lambda\right]
\end{aligned}
$$

where $k_{1}$ is an integral gain. Figure 6 shows the response of the system (27) to the different controllers. Tracking has been improved and still no extensive knowledge about the system is used.

Using measurements of the angular accelerations for control of the attitude rate naturally gives rise to the question what would happen in the presence of sensor noise. Therefore the measured signals are made noisy using band-limited white noise. Since the angular rate sensors (gyro's) have been researched and developed a lot longer than the commercially-of-the-shelf available angular acceleration sensors, the latter has been given a noise power 10 times larger than the former. The results are shown in Figure 7. Clearly the results of both backstepping and SBB controllers are in the same order of magnitude. Therefore the influence of the angular accelerations noise on the system response is small.

Once again uncertainty is added to the model. Most uncertainty in this system lies in the values of the aerodynamic coefficients. In this case only $1 \%$ is added to the control derivatives $C_{l_{\delta_{a}}}, C_{m_{\delta_{e}}}$ and $C_{n_{\delta_{r}}}$. The results are shown in Figure 8. The conventional controller becomes instable even with such a small change in the parameters. The sensor based backstepping controller is not influenced by the changes.

\section{Conclusions and Recommendations}

This paper presented a backstepping controller, based on singular perturbation theory, that uses measurement data rather than model knowledge. Good tracking performance with or without uncertainty is not only obtained for simple systems, but also for a complex system, based on the equations of motion of an aircraft. The influence of measurement noise was shown to be small and comparable to the results obtained using conventional backstepping. Since the sensor based controller is based on Lyapunov functions, stability of the controlled system is guaranteed. With the advantage over conventional backstepping that model uncertainty plays only a minor role, the sensor based backstepping control law may very well be an important step towards certification of an advanced control law.

Before real life application is an option, however, the influence of time-delays needs to be investigated. Furthermore, the forces were neglected in the calculation of the rotational rate of the aerodynamic reference frame. Investigation is needed whether this additional dynamics will cause problems for the sensor based control system.

\section{References}

\footnotetext{
${ }^{1}$ Calise, A. and Rysdyk, R., "Nonlinear adaptive flight control using neural networks," Control Systems Magazine, IEEE, Vol. 18, No. 6, Dec 1998, pp. 14-25.

${ }^{2}$ Lane, S. H. and Stengel, R. F., "Flight control design using non-linear inverse dynamics," Automatica, Vol. 24, No. 4, July 1988, pp. 471-483.

${ }^{3}$ Khalil, H. K., Nonlinear Systems, Prentice Hall, 3rd edition, 2002.

${ }^{4}$ Krstić, M., Kanellakopoulos, I., and Kokotović, P. V., Nonlinear and Adaptive Control Design, John Wiley \& Sons, Inc. New York, NY, USA, 1995.

${ }^{5} \mathrm{RTCA}$, Software Considerations in Airborne Systems and Equipment Certification, DO-178B, Radio Technical Commission for Aeronautics Inc., 1994.

${ }^{6}$ Sonneveldt, L., Adaptive Backstepping Flight Control for Modern Fighter Aircraft, Ph.D. thesis, Delft University of Technology, 2010.

${ }^{7}$ Jacklin, S. A., "Closing the Certification Gaps in Adaptive Flight Control Software," Guidance, Navigation, and Control Conference, Honolulu, Hawaii, Aug 18-21, 2008, 2008.

${ }^{8}$ Hovakimyan, N., Lavretsky, E., and Sasane, A., "Dynamic Inversion for Nonaffine-In-Control Systems Via Time-scale Separation Part I," Journal of Dynamical and Control Systems, Vol. 13, No. 4, October 2007, pp. 451465.

${ }^{9}$ Bacon, B. J., Ostroff, A. J., and Joshi, S. M., "Reconfigurable NDI Controller Using Inertial Sensor Failure Detection \& Isolation," IEEE Transactions on Aerospace and Electronic Systems, Vol. 37, No. 4, October 2001, pp. 1373-1383.
} 

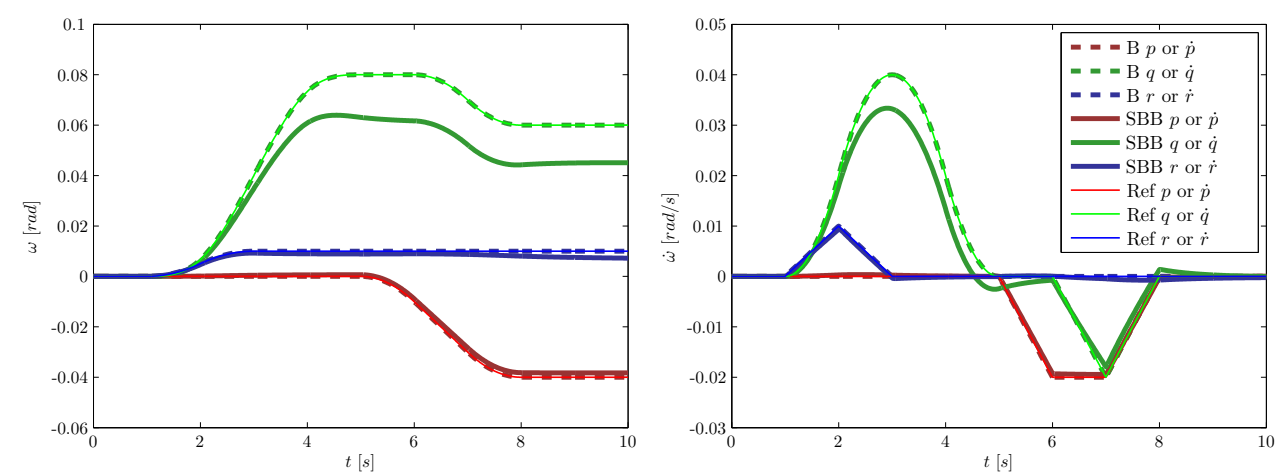

Figure 5. Response of system (27) using a backstepping $\left(c_{4}=1\right)$ and a sensor based backstepping controller $\left(c_{5}=1, \epsilon=0.1\right)$.
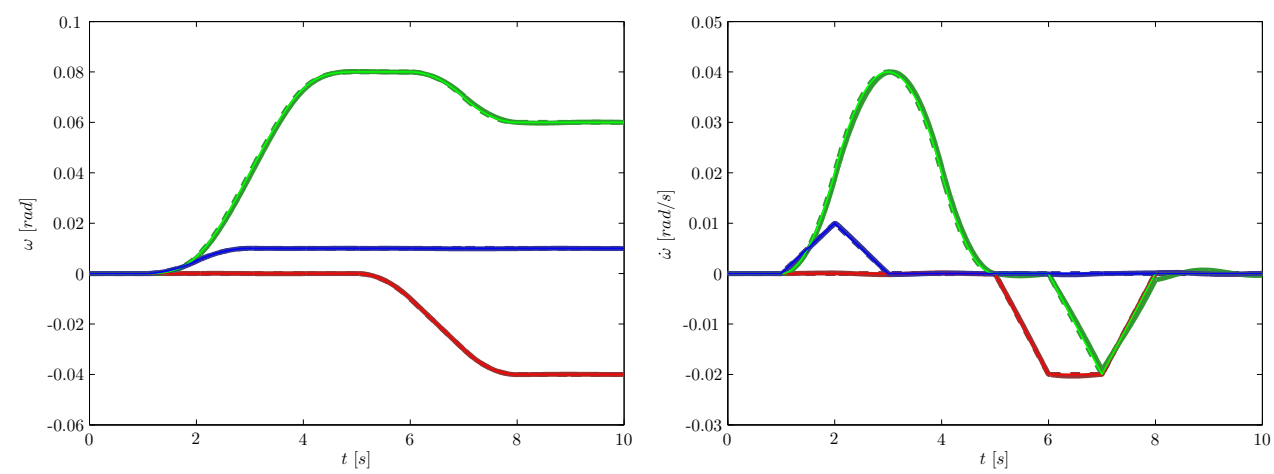

Figure 6. Response of system (27) using a backstepping $\left(c_{4}=1\right)$ and a sensor based backstepping controller with integral gain $\left(c_{5}=1, \epsilon=0.1, k_{1}=10\right)$.
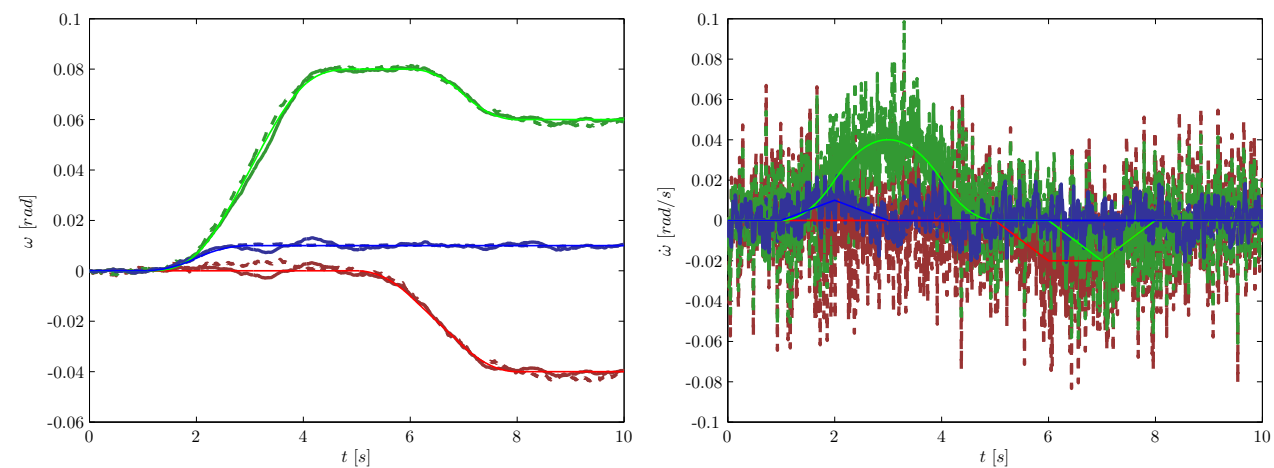

Figure 7. Response of system (27) using a backstepping $\left(c_{4}=1\right)$ and a sensor based backstepping controller $\left(c_{5}=1, \epsilon=0.1, k_{1}=10\right)$ in the presence of sensor noise.
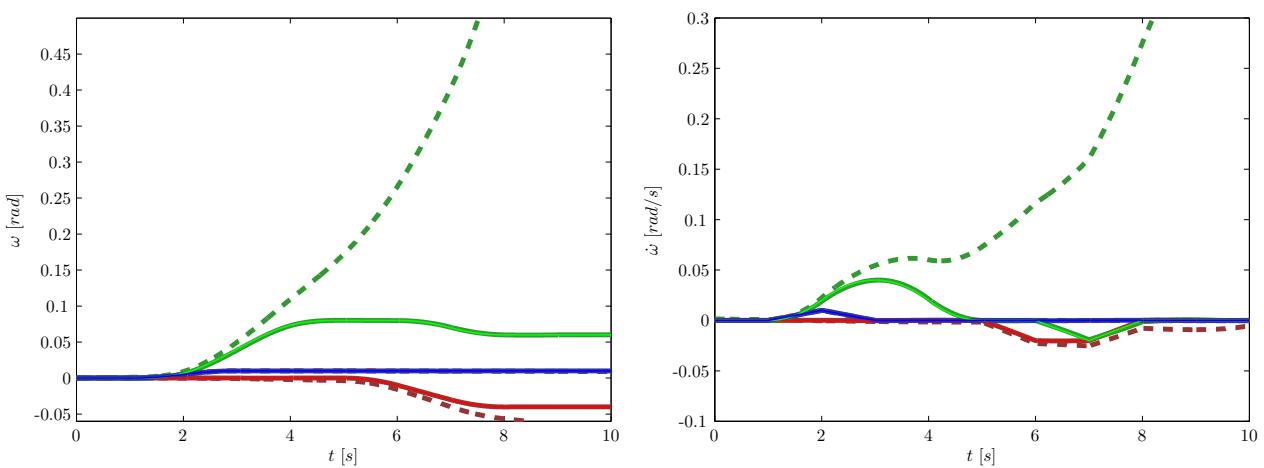

Figure 8. Response of system (27) using a backstepping $\left(c_{4}=1\right)$ and a sensor based backstepping controller $\left(c_{5}=1, \epsilon=0.1, k_{1}=10\right)$ in the presence of uncertainty in the control derivatives. 
${ }^{10}$ Tikhonov, A. N., "Systems of Differential Equations Containing Small Parameters in the Derivatives," Mathematical Sb. (N.S.), Vol. 31(73), No. 3, 1952, pp. 575-586, (in Russian).

${ }^{11}$ Murray, R. M., Li, Z., and Sastry, S. S., A Mathematical Introduction to Robotic Manipulation, CRC Press, 1994.

${ }^{12}$ Mulder, J. A., van Staveren, W., van der Vaart, J. C., and de Weerdt, E., "Flight Dynamics, Lecture Notes AE3-302," Tech. rep., Delft University of Technology, 2006.

${ }^{13}$ Lewis, B. L. and Stevens, F. L., Aircraft Control and Simulation, John Wiley and Sons, 1992. 